\title{
Experimental Evidence of Absolute and Convective Instabilities in Optics
}

\author{
Eric Louvergneaux, Christophe Szwaj, Gonzague Agez, Pierre Glorieux, and Majid Taki \\ Laboratoire de Physique des Lasers, Atomes et Molécules, UMR CNRS 8523, Centre d'Études et de Recherches Lasers et \\ Applications, Université des Sciences et Technologies de Lille, 59655 Villeneuve d'Ascq Cedex, France \\ (Received 20 June 2003; revised manuscript received 26 September 2003; published 27 January 2004)
}

\begin{abstract}
Experimental evidence of convective and absolute instabilities in a nonlinear optical system is given. In optics, the presence of spatial nonuniformities brings in additional complexity. Hence, signatures characterizing these two regimes are derived based on analytical and numerical investigations. The corresponding noise-sustained and dynamical patterns are observed experimentally in a liquid crystal layer subjected to a laser beam with tilted feedback.
\end{abstract}

DOI: 10.1103/PhysRevLett.92.043901

Spatiotemporal instabilities have been predicted in many different nonlinear systems. They arise from the spontaneous symmetry breaking which leads to pattern formation. The phenomenology of these instabilities is completely modified as soon as a drift is present in the system (breaking of the reflection symmetry $\vec{r} \rightarrow-\vec{r}$ ). Indeed, transverse propagating waves rise at the onset. One has then to deal not only with the growth of disturbances, but also with their propagation. As a result, if an instability occurs in these systems, it can be either convective or absolute. The concepts of convective instability (CI) and absolute instability (AI) were first developed in the context of plasma physics [1], and later successfully used in hydrodynamics [2]. In the convective regime, an initial local disturbance exponentially grows but is simultaneously advected away so that the system returns locally to the initial homogeneous equilibrium state. In contrast, disturbances in the absolute regime grow locally fighting the drift upstream so that the system reaches a patterned state. The convective regime, where no pattern is expected, can, however, show structures if noise is present in the system. Then, macroscopic noise-sustained structures (NSS) result from the selective spatial amplification of the continuous perturbations produced by the microscopic noise source.

So far convective instabilities leading to noisesustained structures were theoretically predicted in many areas such as open flows [3], optics [4], traffic flow [5], crystal growth [6], etc. They were obtained experimentally in hydrodynamics [7-9] but still wait to be observed in extended optical systems $[10,11]$. In the latter field, the question of the existence of such instabilities (CI, NSS) in real systems, i.e., surviving the limitations of real devices, is open. Namely, in most optical systems control parameters are space dependent (Gaussian pump profile, curved mirrors). Thus, the characterization criteria established by theoretical works for ideal systems, i.e., with a uniform incident plane wave and space independent parameters, cannot be applied directly [12] and have to be improved.
PACS numbers: 42.65.Sf, 05.40.Ca, 05.45.-a, 47.54.+r

The purpose of this Letter is to evidence theoretically and experimentally the convective instability in optics, but also more generally in nonideal systems. The system considered here is a nematic liquid crystal layer irradiated by a laser beam and inserted in a feedback loop with a tilted mirror [13]. We emphasize that our real system (i) is noisy with an inherent noise source stemming from thermal fluctuations of the liquid crystal and (ii) possesses control parameters that are space dependent due to the Gaussian incident beam. We focus here on the onedimensional (1D) case. We derive, first, analytical thresholds for convective and absolute instabilities in the ideal situation where noise is neglected and the incident wave is a plane wave. Then, from numerical simulations carried out for the experimental conditions, we find signatures for these two regimes showing that the scenario of these instabilities still remains with these specific features. Finally, by comparison between the CI and AI signatures of these numerical simulations and of experimental recordings, we prove that CI characterized by NSS are, indeed, experimentally observable in real systems.

Experiments have been realized on a feedback optical system (Fig. 1) well described in the framework of a model introduced first by Akhmanov et al. [14] and later adapted by Firth and d'Alessandro [15]. Here this model is slightly modified to account for the input field spatial variation and for the noise. In the $1 \mathrm{D}$ case it reads

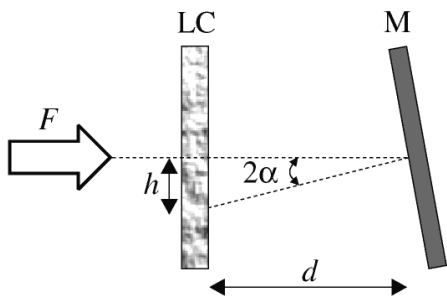

FIG. 1. Schematic sketch of the experimental setup. LC, liquid crystal layer; $\mathrm{M}$, feedback mirror; $F$, input optical field; $\alpha$, mirror tilt angle; $d$, feedback length. 


$$
\begin{gathered}
-\nabla_{\perp}^{2} n+\frac{\partial n}{\partial t}+n=|F|^{2}+|B|^{2}+\sqrt{\epsilon} \xi(x, t), \\
B(x, t)=\sqrt{R} F_{0} e^{i \sigma \nabla_{\perp}^{2}}\left(e^{i \chi n(x+h, t)} g(x)\right),
\end{gathered}
$$

where $n(x, t)$ stands for the refractive index of the nonlinear nematic LC layer, $t$ and $x$ are the time and space variables scaled with respect to the relaxation time $\tau$ and the diffusion length $l_{D}, h$ represents the lateral shift due to the tilted mirror as indicated in Fig. 1 , and $R$ is its reflectivity. We have set $\sigma=d / k_{0} l_{D}^{2}$ where $d$ is the slicemirror distance and $k_{0}$ is the optical wave number of the field. $\xi(x, t)$ describes a Gaussian stochastic process of zero mean and correlation $\left\langle\xi(x, t) \xi\left(x^{\prime}, t^{\prime}\right)\right\rangle=$ $\delta\left(x-x^{\prime}\right) \delta\left(t-t^{\prime}\right)$. The level of noise is controlled by the parameter $\epsilon$, which is purely phenomenological. $F$ is the forward input optical field, and its transverse profile is accounted for by using $F(x)=F_{0} g(x)$, with $g(x)=$ $\exp \left(-x^{2} / w^{2}\right)$ for a Gaussian pump beam of radius $w$ and $g=1$ for the uniform (plane wave) case. $B$ is the backward optical field [15]. The Kerr effect is parametrized by $\chi$ which is positive (negative) for a focusing (defocusing) medium.

Starting from the above equations, in the plane wave approximation $[g(x)=1]$ and in the absence of noise $(\epsilon=0)$, we can perform a linear stability analysis that provides us with the convective and absolute thresholds in the presence of a feedback mirror tilt. Assuming perturbations of the stationary state $n_{0}=F_{0}^{2}(1+R)$ in the form $\Delta n \sim \exp (i k x-\lambda t)$, we obtain the following dispersion relation:

$$
\lambda=1+k^{2}-\mu \sin \left(\sigma k^{2}\right) \exp (i k h),
$$

where $\mu=2 R F_{0}^{2}|\chi|$. The above expression shows that the presence of $h$ leads to a complex dispersion relation. This means that, in addition to the classical temporal instabilities ( $k$ real and $\lambda$ complex), there are also spatial amplifications ( $k$ complex). Near onset of the instability, Eq. (3) may be approximated [16] as

$$
\begin{aligned}
\lambda^{\mathrm{app}}= & \lambda_{c}+\left.\left(\frac{\partial \lambda}{\partial \mu}\right)\right|_{c}\left(\mu-\mu_{c}\right)+\left.\left(\frac{\partial \lambda}{\partial k}\right)\right|_{c}\left(k-k_{c}\right) \\
& +\left.\frac{1}{2}\left(\frac{\partial^{2} \lambda}{\partial^{2} k}\right)\right|_{c}\left(k-k_{c}\right)^{2}
\end{aligned}
$$

where the derivatives are evaluated at $\mu_{c}$ and $k_{c}$ that are the critical values given by Eq. (3) and correspond to $\mu_{\text {conv }}$ and $k_{\text {conv }}$ (in our notations), and $\lambda_{c}=\lambda\left(k_{c}, \mu_{c}\right)$. After simplifications, we find $\partial \lambda /\left.\partial \mu\right|_{c}=-\sin \left(\sigma k_{c}^{2}\right) \times$ $\exp \left(i h k_{c}\right), \quad \partial \lambda /\left.\partial k\right|_{c}=-i\left(1+k_{c}^{2}\right)\left[h+2 \sigma k_{c} \tan \left(h k_{c}\right) /\right.$ $\left.\tan \left(\sigma k_{c}^{2}\right)\right], \quad$ and $\quad \partial^{2} \lambda /\left.\partial^{2} k\right|_{c}=\left(1+k_{c}^{2}\right)\left\{4 \sigma^{2} k_{c}^{2}+h^{2}+\right.$ $\left.\tan \left(h k_{c}\right)\left[4 \sigma k_{c} / \tan \left(\sigma k_{c}^{2}\right)-h / k_{c}\right]\right\}+i\left\{\left(1+k_{c}^{2}\right) \tan \left(h k_{c}\right) \times\right.$ $\left.\left[4 \sigma^{2} k_{c}^{2}-2 \sigma / \tan \left(\sigma k_{c}^{2}\right)-h^{2}\right]-4 h k_{c}\right\}$. Note that these expressions are obtained without any restriction on the value of $h$. The key feature is the presence of a group velocity [imaginary part of the third term in Eq. (4)] which vanishes for $h=0$, meaning that a convective regime may exist if $h \neq 0$.

043901-2
The convective threshold $\mu_{\text {conv }}$ is obtained by canceling $\operatorname{Re}(\partial \lambda / \partial k)_{k_{c} \text { real }}$ in Eq. (4), whereas the absolute instability threshold $\mu_{\mathrm{abs}}$ is given by the saddle point method. It states that the absolute threshold is such that $\operatorname{Re}\left(\lambda\left(k_{s}\right)\right)=0$ where $k_{s}$ is a complex saddle point satisfying $\nabla_{k} \lambda(k)=0$ and $\operatorname{Re}\left[\nabla_{k}^{2} \lambda(k)\right] \geq 0$ (see Refs. [2,17-19] for details). The thresholds are plotted in Fig. 2 versus $h$. As can be seen from the figure, there exists a region $\mu_{\text {conv }}<\mu<\mu_{\text {abs }}$ where a convective regime is expected.

The analytical approach proves firmly the existence of $\mathrm{CI}$ and $\mathrm{AI}$ in the ideal system; in addition, it allows one to determine the parameter domain for $h$ and $\chi \sigma$ that maximizes the convective region. However, the experimental conditions differ mainly by the presence of a Gaussian pump beam and noise that may affect drastically the dynamics [20,21]. Fortunately, numerical investigations performed in the parameter range of our experiments show that these experimental features do not modify qualitatively the scenario of CI and AI. In what follows, we describe (i) the numerical test for demonstrating the CI persistence in the presence of a Gaussian pumping without noise, (ii) the signature found numerically showing that the addition of noise does not affect the CI and AI threshold values (determined without noise) and their scenario, and (iii) the role of this signature to evidence the CI and AI regimes in the experiments.

In a first step we check numerically the influence of a Gaussian input field on the existence of CI. In that purpose, we analyze (in the absence of noise) the spatiotemporal evolution of a small perturbation applied initially on the top of the Gaussian beam for increasing values of the parameter $\mu$ [Figs. 3(a)-3(c)], and identify three different regimes. Namely, a homogeneous regime where the perturbation is linearly damped [Fig. 3(a)]. A convective regime, the perturbation is amplified and advected away. The system returns to the basic state for long times [Fig. 3(b)]. An absolute regime, the pattern invades all the available space [Fig. 3(c)]. The transitions between these regimes set the values of the convective and absolute thresholds (second row of Table I). Note that, as mentioned above, these values differ slightly from the analytical predictions obtained in the ideal case $(\dagger$ in Table I). If we further reduce the Gaussian width, the

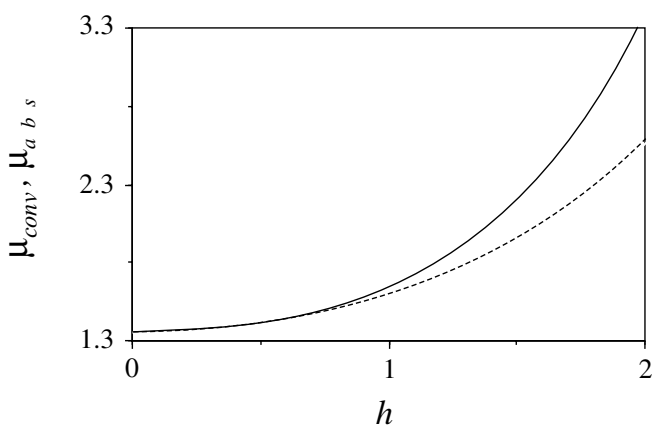

FIG. 2. Absolute (solid line) and convective (dashed line) thresholds versus $h$ for $\sigma=4.23$ (as in experiments).

043901-2 

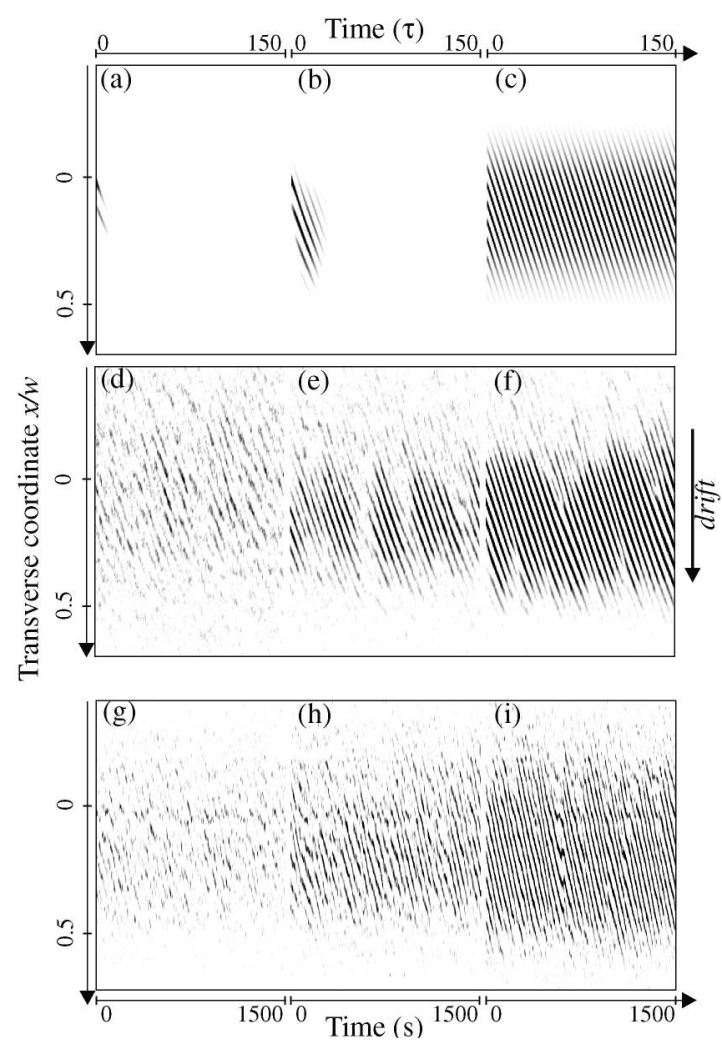

FIG. 3. (a)-(c) Numerical simulations of the evolution of a perturbation applied at $(x=0, t=0)$, in case of a Gaussian pump beam without noise. (d)-(f) Numerical simulations of the "patterns" obtained for the same Gaussian input beam but in the presence of noise. (g)-(i) Corresponding experimental observations. Pictures (a),(d),(g) correspond to a regime below CI threshold, (b),(e),(h) to CI, and (c),(f),(i) to AI. The parameters are $w=140, \quad \sigma=4.23, h=1.3, \quad \epsilon=10^{-2}$. (a),(d) $F_{0}^{2}=0.98 ;$ (b),(e) $F_{0}^{2}=1.1$; (c),(f) $F_{0}^{2}=1.21$. (g)(i) $d=5 \mathrm{~mm}, \quad w=1400 \mu \mathrm{m}, \quad \alpha \simeq 1.3 \mathrm{mrad}, \quad h=1.3 l_{D}$. (g) $I_{0}=95 \mathrm{~W} / \mathrm{cm}^{2}$, (h) $I_{0}=110 \mathrm{~W} / \mathrm{cm}^{2}$, (i) $I_{0}=128 \mathrm{~W} / \mathrm{cm}^{2}$.

separation between the two thresholds increases more. So, the addition of a Gaussian profile does not affect the existence of a convective region-only its domain of existence varies with the pump width.

In the second step we take into account noise $(\epsilon \neq 0)$ in Eqs. (1) and (2). Numerical integrations for the same parameters as previously are carried out to obtain the realistic "noisy" patterns [Figs. 3(d)-3(f)] that are expected experimentally. Below the convective threshold (determined without noise), they depict an intermittent

TABLE I. Comparison of the numerical values of the convective and absolute thresholds obtained from ( $\dagger$ ) the local perturbation method and $(\diamond)$ the edge detection $\bar{u}_{f} \cdot w=140$, $\sigma=4.23, h=1.3, \epsilon=10^{-2} . \Delta\left(F_{0}^{2}\right)$ is the uncertainty.

\begin{tabular}{lllc}
\hline \hline & $F_{0 \text { conv }}^{2}$ & $F_{0 \text { abs }}^{2}$ & $\Delta\left(F_{0}^{2}\right)$ \\
\hline Flat profile, noise $(\equiv$ analytic) $\dagger$ & 0.998 & 1.073 & $\ldots$ \\
Gaussian profile, no noise $\dagger$ & 1.05 & 1.16 & 0.02 \\
Gaussian profile and noise $\diamond$ & 1.05 & 1.16 & 0.02 \\
\hline
\end{tabular}

043901-3 pattern of very small amplitude and with a random spatial phase [Fig. 3(d)]. It is associated with a precursor, induced by noise [22], of the pattern that emerges at threshold. In the convective regime [Fig. 3(e)], the perturbations are amplified and advected away, but since they appear recursively they lead to NSS. Above the absolute threshold (determined without noise, i.e., 1.16) the domain of the roll pattern extends upstream and invades all the available region [Fig. 3(f)]. Although the pump profile is symmetric with respect to its center (position $x=0$ ), the patterns are located to the bottom. This is a direct consequence of the amplification and advection. As a result, drifting rolls are observed for both unstable regimes CI and AI [Fig. 3(e) and 3(f)]. However, these two regimes do not differ sufficiently to be distinguished without any ambiguity from the figure. In order to evidence the CI and NSS regimes, we look for a signature of the convective and absolute regimes based on the evolution of the width of the pattern region versus $\mu$ and more precisely of the evolution of its upstream edge $\left(\bar{u}_{f}\right)$ which is the most relevant. It is defined as the mean location where the roll amplitude is $1 / 10$ of its maximum (at the corresponding $\mu$ ). Indeed, Fig. 4(a) reveals that $\bar{u}_{f}$ displays three different evolutions characterized by two critical values of $F_{0}^{2}(\diamond$ in Table I) corresponding to the two transitions in the evolution of $\bar{u}_{f}$ [vertical dashed lines in Fig. 4(a)]. Comparison between these critical values and the numerical evaluations of the CI-AI thresholds ( $\dagger$ second row in Table I) previously found for a Gaussian pumping without noise are in excellent agreement. One may conclude that the first breaking in the curve of $\bar{u}_{f}$ occurring at $F_{0}^{2}=1.05$ (left dashed vertical line) corresponds to the convective instability threshold while that observed at $F_{0}^{2}=1.16$ (right dashed vertical line) is associated with the onset of the absolute instability. This means that the three distinct evolutions of $\bar{u}_{f}$ correspond to the three different regimes of below $\mathrm{CI}$ threshold, CI, and AI (respectively, regions labeled $\mathrm{H}$, $\mathrm{C}$, and $\mathrm{A}$ in Fig. 4). The above quantitative comparison demonstrates that the addition of a realistic level of noise does not change the scenario of the instabilities. Finally,

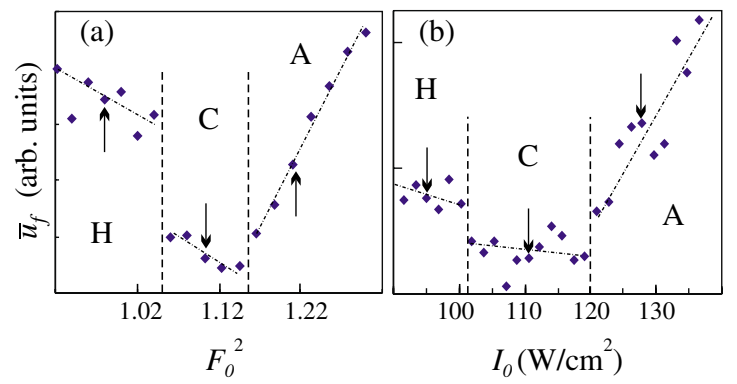

FIG. 4 (color online). Evolution of $\bar{u}_{f}$ versus the incident intensity. Left: numerical simulations; right: experiments. Same parameters as Fig. 3. H, C, and A refer to the homogeneous, convective, and absolute regimes, respectively. The arrows correspond to the diagrams of Fig. 3. 
the jumps in the slope evolution of $\bar{u}_{f}$ provide a criterion for evidencing the CI regime in the realistic conditions. Note that the study of temporal Fourier transform of the intensity at a space location $[4,23]$ confirms the existence of the two regimes (CI and $\mathrm{AI})$.

Corresponding experiments have been performed on the setup already described in Refs. [20,22], with the essential difference of having a feedback mirror tilted by an angle $\alpha$ (see Fig. 1). As the power of the input laser beam is increased, we observe experimentally [Figs. 3(g)3(i)] three qualitatively different spatiotemporal behaviors that are similar to those of numerical simulations [Figs. 3(d)-3(f)]. For low input intensities, the output beam intensity shows erratic rolls that appear randomly in time and space with short time duration [Fig. 3(g)]. When increasing the input intensity, drifting "rolls" are observed that never die out and have a spread width increasing with $I_{0}$ [Figs. 3(h) and 3(i)]. The inspection of the $\bar{u}_{f}$ evolution evaluated from the experimental recordings [Fig. 4(b)] shows clearly three distinct regimes that mimic those of the realistic numerical simulations [Fig. 4(a)]. By comparison, we conclude the existence of a convective regime [C region in Fig. 4(b)] and locate approximately the two thresholds. The agreement between the simulations and the experiments is quantitatively good with a ratio of the absolute to convective "thresholds" of about, respectively, 1.1 and 1.07.

To summarize, we have demonstrated that noisesustained rolls associated with the convectively unstable regime survive in a real system with parameters slowly varying in space. The existence of a CI regime highlights the crucial role of noise source in the dynamics of patterns. Indeed, these structures result from the amplification of perturbations by deterministic dynamics. This contrasts with other noise effects as, for instance, the noisy precursors corresponding to weakly damped fluctuations observed in the linear stable regime [22]. The necessary condition for the occurrence of the convective regime is the reflection symmetry breaking which is produced here by feedback mirror tilt, thus producing a drift velocity. Dynamical rolls as due to the absolute instability have also been observed beyond the CI domain. They are self-sustained by the nonlinear dynamics. Experiments and simulations show that the best indicator of the nature of the instabilities is provided by the evolution of the boundaries of the patterned region. In spite of the presence of noise, this evolution exhibits clear thresholds for convective and absolute instabilities. The statistics and temporal Fourier transform confirm that there exists a sizable domain of convective instabilities between the unpatterned and the fully patterned states.

We thank M. Tlidi, P. Collet, P. L. Ramazza, M. San Miguel, and R. Zambrini for useful discussions. The Laboratoire de Physique des Lasers, Atomes et Molecules is Unite de Recherche Mixte du CNRS. The Centre d'Études et de Recherches Lasers et Applications is supported by the Ministère chargé de la Recherche, the
Région Nord-Pas de Calais, and the Fonds Européen de Développement Économique des Régions.

[1] R. J. Briggs, Electron-Stream Interaction with Plasmas (MIT Press, Cambridge, MA, 1964).

[2] P. Huerre and P. A. Monkewitz, Annu. Rev. Fluid Mech. 22, 473 (1990).

[3] A. Couairon and J. M. Chomaz, Phys. Rev. Lett. 79, 2666 (1997); H. R. Brand, R. J. Deissler, and G. Ahlers, Phys. Rev. A 43, 4262 (1991).

[4] M. Santagiustina, P. Colet, M. San Miguel, and D. Walgraef, Phys. Rev. Lett. 79, 3633 (1997).

[5] N. Mitarai and H. Nakanishi, Phys. Rev. Lett. 85, 1766 (2000).

[6] N. Israeli, D. Kandel, M. F. Schatz, and A. Zangwill, Surf. Sci. 494, L735 (2001).

[7] P. Büchel and M. Lucke, Phys. Rev. E 61, 3793 (2000); X. Nicolas, A. Mojtabi, and J. K. Platten, Phys. Fluids 9, 337 (1997).

[8] P. Gondret, P. Ern, L. Meignin, and M. Rabaud, Phys. Rev. Lett. 82, 1442 (1999).

[9] L. Pastur, M.T. Westra, and W. van de Water, Physica (Amsterdam) 174D, 71 (2003).

[10] M. Taki, N. Ouarzazi, H. Ward, and P. Glorieux, J. Opt. Soc. Am. B 17, 997 (2000); H. Ward, M. Taki, and P. Glorieux, Opt. Lett. 27, 348 (2002).

[11] G. Izùs, M. Santagiustina, M. San Miguel, and P. Colet, J. Opt. Soc. Am. B 16, 1592 (1999); M. Taki, M. San Miguel, and M. Santagiustina, Phys. Rev. E 61, 2133 (2000).

[12] M. N. Ouarzazi, P. A. Bois, and M. Taki, Phys. Rev. A 53, 4408 (1996).

[13] P. L. Ramazza, S. Ducci, and F.T. Arecchi, Phys. Rev. Lett. 81, 4128 (1998); S. Ducci, P. L. Ramazza, W. González-Viñas, and F.T. Arecchi, Phys. Rev. Lett. 83, 5210 (1999).

[14] S. A. Akhmanov, M. A. Vorontsov, and V. Yu. Ivanov, JETP Lett. 47, 707 (1988); S. A. Akhmanov, M. A. Vorontsov, V.Yu. Ivanov, A.V. Larichev, and N. I. Zheleznykh, J. Opt. Soc. Am. B 9, 78 (1992).

[15] W. J. Firth, J. Mod. Opt. 37, 151 (1990); G. D’Alessandro and W. J. Firth, Phys. Rev. Lett. 66, 2597 (1991).

[16] A. D. D. Craik, Proc. R. Soc. London, Ser. A 373, 457 (1981); R. Tagg, W. S. Edwards, and H. L. Swinney, Phys. Rev. A 42, 831 (1990).

[17] M. Santagiustina, P. Colet, M. San Miguel, and D. Walgraef, Phys. Rev. E 58, 3843 (1998).

[18] H. Ward, M. N. Ouarzazi, M. Taki, and P. Glorieux, Eur. Phys. J. D 3, 275 (1998).

[19] H. Ward, M. N. Ouarzazi, M. Taki, and P. Glorieux, Phys. Rev. E 63, 016604 (2001).

[20] E. Louvergneaux, Phys. Rev. Lett. 87, 244501 (2001).

[21] M. San Miguel and R. Toral, Instabilities and Nonequilibrium Structures VI (Kluwer Academic Publishers, Dordrecht, 2000), Pt. I.

[22] G. Agez, C. Szwaj, E. Louvergneaux, and P. Glorieux, Phys. Rev. A 66, 063805 (2002).

[23] K. L. Babcock, G. Ahlers, and D. S. Cannell, Phys. Rev. Lett. 67, 3388 (1991). 